\title{
Fluid-Structure Interaction by the Spectral Element Method
}

\author{
N. Bodard ${ }^{1}$ and M. O. Deville ${ }^{1}$
}

Received October 20, 2004; accepted (in revised form) October 10, 2005; Published online March 13, 2006

\begin{abstract}
Viscous fluid-structure interaction is treated with an arbitrary LagrangianEulerian formulation. The spatial discretization is performed by the spectral element method for the fluid part where the Navier-Stokes equations are integrated and in the solid part where transient linear elasticity is described by the Navier equations. Time marching algorithms are second-order accurate in time in both the fluid and the solid. The algorithm is applied to the flow in a plane channel partially obstructed by a solid component able to move under the action of the fluid flow.
\end{abstract}

KEY WORDS: Spectral element; fluid-structure interaction; ALE.

\section{INTRODUCTION}

Fluid-structure interaction (FSI) has been studied for several decades in many applications as aerodynamics, hydrodynamics or haemodynamics $[1,2]$. Major algorithmic developments were achieved like the arbitrary Lagrangian-Eulerian (ALE) formulation [3], the mesh adaptation [4] or the fictitious domain techniques [5,6]. A fixed point method can be used [1, 2, 7] to improve the stability. The FSI has been treated mostly by finite volume or finite element methods. We investigate a fluid-structure algorithm to assess the feasibility of the spectral element method in this context following the methodology developed by Ho for free surface flows [8]. We tackle the problem by the spectral element method (SEM) for the fluid and solid parts. The state of the art for SEM fluid flow problems is given in [9] while for elasticity the reader is referred to the papers [10, 11]. In the long run we want to simulate the flow through an aortic valve. To this

\footnotetext{
${ }^{1}$ Ecole Polytechnique Fédérale de Lausanne, Laboratory of Computational Engineering, CH-1015 Lausanne, Switzerland.
} 


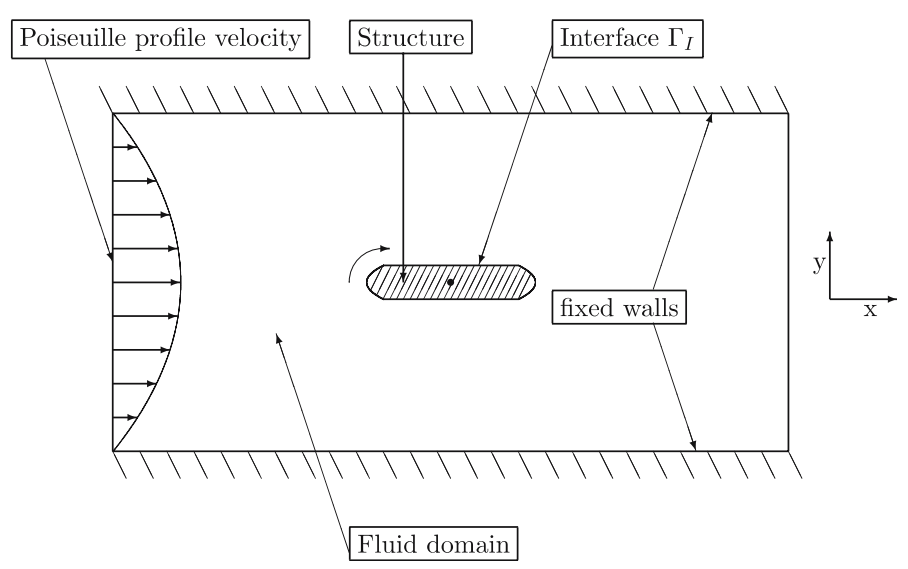

Fig. 1. Geometry of the problem.

end, Fig. 1 shows the relevant geometry. The fluid flows in the $x$ direction into a plane channel with fixed horizontal walls. Inside the channel we observe a solid object which can turn around its fixed center of mass.

To solve this viscous fluid-structure interaction we will use the ALE technique [12]. From the methodological point of view, the problem is set up in three stages. First, for the fluid model we will consider an incompressible viscous Newtonian fluid and the Navier-Stokes equations are used. Second, for the solid model, we will solve the dynamics equations for an elastic structure. We assume that the coupled problem is isothermal. In the third step, the interaction equation between the fluid and the structure leads to special Neumann and Dirichlet conditions at the fluidstructure boundary interface. Due to the fact the fluid equations are classically expressed in the Eulerian framework while the structure equations are given in the Lagrangian framework, the strategy consists in combining both previous points of view in the ALE formulation to match the nodes of the structure and the fluid at the interface. In the last part, we will present the numerical results.

\section{NAVIER-STOKES PROBLEM}

\subsection{Equations in a Moving Domain: The Mathematical Model}

The incompressible Navier-Stokes equations in the fluid domain $\Omega^{f}$ are

$$
\rho_{f} \frac{D \mathbf{v}}{D t}=\nabla \cdot \boldsymbol{\sigma}_{f}+\rho_{f} \mathbf{b}_{f}
$$


and the incompressibility constraint

$$
\nabla \cdot \mathbf{v}=0
$$

where

$$
\boldsymbol{\sigma}_{f}=-p \mathbf{I}+\mu_{f}\left(\nabla \mathbf{v}+(\nabla \mathbf{v})^{T}\right)=-p \mathbf{I}+2 \mu_{f} \mathbf{D}(\mathbf{v})
$$

$D / D t$ is the material time derivative, $p$ the pressure, I the identity tensor, $\mathbf{v}$ the velocity vector, $T$ indicates the transpose, $\mu_{f}$ is the dynamic shear viscosity, $\mathbf{b}_{f}$ the body force, $\mathbf{D}$ the rate-of-deformation tensor and $\rho_{f}$ the fluid volumetric mass. We also note $\Gamma_{D}$ (respectively, $\Gamma_{N}$ ) the boundary of $\Omega^{f}$ where Dirichlet (respectively, Neumann) conditions are applied.

On $\Gamma_{D}$ we have

$$
\mathbf{v}=\left.\overline{\mathbf{v}}\right|_{\Gamma_{D}}
$$

with $\left.\overline{\mathbf{v}}\right|_{\Gamma_{D}}$ given on $\Gamma_{D}$. In the particular case of (Fig. 1), we can divide $\Gamma_{D}$ such as

$$
\Gamma_{D}=\Gamma_{D}^{i n} \cup \Gamma_{D}^{\text {wall }} \cup \Gamma_{I}
$$

$\Gamma_{I}$ denotes the fluid-structure interface, $\Gamma_{D}^{i n}$ the inflow boundary with a velocity Poiseuille profile and $\Gamma_{D}^{\text {wall }}$ the boundary part of the external fixed walls. At the outflow section, we impose a stress-free boundary condition.

In the ALE framework [13, 14], we introduce a reference configuration $\Omega_{0}^{f}$ with $\mathbf{X}$ coordinates. The domain $\Omega_{t}^{f}$ is the actual configuration with $\boldsymbol{x}$ coordinates. Hence, we define the mapping $A_{t}$

$$
\begin{aligned}
& A_{t}: \Omega_{0}^{f} \subset \mathbf{R}^{d} \rightarrow \Omega_{t}^{f} \subset \mathbf{R}^{d}, \\
& \mathbf{X} \rightarrow \boldsymbol{x}(\mathbf{X}, t)=A_{t}(\mathbf{X}),
\end{aligned}
$$

$\mathrm{d}$ is the space dimension and $\mathrm{t}$ the time in the interval $I=\left[t_{0}, T\right]$.

The ALE velocity $\mathbf{w}$ is defined

$$
\mathbf{w}=\left.\frac{\partial A_{t}}{\partial t}(\mathbf{X})\right|_{\mathbf{X}}=\left.\frac{\partial x(\mathbf{X}, t)}{\partial t}\right|_{\mathbf{X}} .
$$

The ALE time derivative of the velocity $\mathbf{v}$ is

$$
\left.\frac{\partial \mathbf{v}}{\partial t}\right|_{\mathbf{X}}=\frac{\partial \mathbf{v}}{\partial t}+\mathbf{w} \cdot \nabla \mathbf{v}
$$


Inserting Eq. (8) in Eq. (1) yields

$$
\rho_{f}\left(\left.\frac{\partial \mathbf{v}}{\partial t}\right|_{\mathbf{X}}+(\mathbf{v}-\mathbf{w}) \cdot \nabla \mathbf{v}\right)=-\nabla p+2 \mu_{f} \nabla \cdot \mathbf{D}(\mathbf{v})+\rho_{f} \mathbf{b}_{f},
$$

Eqs. (9) and (2) are used to solve the fluid flow in our application. The weak formulation to solve Eq. (9) is obtained with the test functions $\hat{\boldsymbol{\Phi}} \in$ $H_{0, D}^{1}\left(\Omega_{0}^{f}\right)$ such as

$$
H_{0, D}^{1}\left(\Omega_{0}^{f}\right)^{d}=\left\{\mathbf{u} \in H^{1}\left(\Omega_{0}^{f}\right)^{d}:\left.\mathbf{u}\right|_{\Gamma_{D}}=0\right\} .
$$

Therefore we want to find $\mathbf{v}$ in the following space

$$
H_{D}^{1}\left(\Omega_{t}^{f}\right)^{d}=\left\{\mathbf{u} \in H^{1}\left(\Omega_{t}^{f}\right)^{d}:\left.\mathbf{u}\right|_{\Gamma_{D}} \text { given }\right\}
$$

The ALE conservative formulation reads:

Find $(\mathbf{v}, p) \in H_{D}^{1}\left(\Omega_{t}^{f}\right)^{d} \times L^{2}\left(\Omega_{t}^{f}\right), t \in I$ such as

$$
\begin{gathered}
\frac{d}{d t} \int_{\Omega_{t}^{f}} \rho_{f} \mathbf{v} \cdot\left(\hat{\boldsymbol{\Phi}} \circ A_{t}^{-1}\right) d \Omega+\int_{\Omega_{t}^{f}} \rho_{f}\{\nabla \cdot(\mathbf{v} \otimes(\mathbf{v}-\mathbf{w}))\} \cdot\left(\hat{\boldsymbol{\Phi}} \circ A_{t}^{-1}\right) d \Omega \\
=\int_{\Omega_{t}^{f}} \rho_{f} p \nabla \cdot\left(\hat{\boldsymbol{\Phi}} \circ A_{t}^{-1}\right)-2 \mu_{f} \nabla \mathbf{v}: \mathbf{D}\left(\hat{\boldsymbol{\Phi}} \circ A_{t}^{-1}\right)+\rho_{f} \mathbf{b}_{f} \cdot\left(\hat{\boldsymbol{\Phi}} \circ A_{t}^{-1}\right) d \Omega, \\
\forall \hat{\boldsymbol{\Phi}} \in H_{0, D}^{1}\left(\Omega_{0}^{f}\right)^{d}, \\
\int_{\Omega_{t}^{f}}(\nabla \cdot \mathbf{v})\left(\hat{\Psi} \circ A_{t}^{-1}\right) d \Omega=0, \quad \forall \hat{\Psi} \in L^{2}\left(\Omega_{0}^{f}\right) .
\end{gathered}
$$

\subsection{Space and Time Discretizations of the Navier-Stokes Equations}

We discretize the ALE conservative formulation (12) cutting $\Omega_{t}^{f}$ in non-overlapping sub-domains $\left(\Omega_{t}^{f}\right)_{k}$ such as $\Omega_{t}^{f}=\cup_{k=1}^{E}\left(\Omega_{t}^{f}\right)_{k}$. For instance, in the 2D case the $\left(\Omega_{t}^{f}\right)_{k}$ are quadrilaterals where we adopt the following mapping [8] linking the physical coordinates $\boldsymbol{x}^{k}=(x, y)^{k} \in$ $\left(\Omega_{t}^{f}\right)_{k}$ into the parent coordinates $\mathbf{r}=(r, s) \in[-1,1]^{2}$. In order to solve the Navier-Stokes equations, the choice of the $P_{N}-P_{N-2}$ discretization [15] avoids the presence of spurious pressure modes. The notation $P_{N}$ represents the space of polynomial of degree less or equal to $N$ in each space direction. The weak formulation of problem (9) and the application of the Galerkin method discretized using a Gauss-Lobatto-Legendre (GLL) quadrature rule for the velocity and a Gauss-Legendre quadrature for the pressure, one obtains using the same symbols as in [9] with the point of view of the ALE formulation

$$
\begin{aligned}
\frac{d}{d t}(\mathbf{M v}) & =-\mathbf{C}(\mathbf{v}, \mathbf{w}) \mathbf{v}-\mathbf{K v}+\mathbf{D}^{T} p+\mathbf{F}, \\
\mathbf{D v} & =0,
\end{aligned}
$$


where $\mathbf{K}$ is the stiffness matrix, $\mathbf{M}$ the mass matrix, $\mathbf{D}$ the discrete divergence, $\mathbf{D}^{T}$ the discrete gradient, $\mathbf{C}$ the convective term depending on the ALE velocity $\mathbf{w}$ and $\mathbf{F}$ the source term.

For the time marching scheme, in a first attempt, a backward differentiation formula of order 2 is used for the linear Stokes operator where the time dependent term is approximated as

$$
\frac{d}{d t}(\mathbf{M v})=\frac{1}{\tau} \mathbf{M}^{n+1}\left(\frac{3}{2} \mathbf{v}^{n+1}-2 \mathbf{v}^{n}+\frac{1}{2} \mathbf{v}^{n-1}\right)
$$

with $\tau$ the time step. The convective term is handled by an extrapolation of order 2 and we use a block LU decomposition scheme with a pressure correction as was proposed in [16, 17].

\section{THE SOLID MECHANICS PROBLEM}

\subsection{The Dynamic Equation}

We introduce the dynamic equation of the linear infinitesimal elasticity. This equation uses the assumption of small deformations. Therefore, the material time derivative is linearized and contains the acceleration written as the second-order partial derivative with respect to time of the displacement denoted by $\mathbf{u}$. We can write

$$
\nabla \cdot \sigma_{s}+\rho_{s} \mathbf{b}_{s}=\rho_{s} \frac{\partial^{2} \mathbf{u}}{\partial t^{2}}
$$

Here, $\sigma_{s}$ is the Cauchy stress tensor, $\mathbf{b}_{s}$ and $\rho_{s}$ the body force and the solid volumetric mass in the structure. Hooke's law gives the constitutive relationship between the stress tensor $\boldsymbol{\sigma}_{s}$ and the strain tensor $\boldsymbol{\varepsilon}$

$$
\sigma_{s}=\lambda \operatorname{tr} \varepsilon \mathbf{I}+2 \mu \varepsilon
$$

where $\lambda$ and $\mu$ are the Lamé coefficients. The strain tensor can be expressed in terms of the displacement by the relation

$$
\boldsymbol{\varepsilon}=\frac{1}{2}\left(\nabla \mathbf{u}+(\nabla \mathbf{u})^{T}\right)
$$

We will rewrite the second-order time problem as a system of first-order differential equations expressed by three fields: the displacement $\mathbf{u}$, the velocity $\dot{\mathbf{u}}=\mathbf{v}$ and the acceleration $\ddot{\mathbf{u}}=\mathbf{a}$, with the convention that the dot denotes the time derivative. 


\subsection{The Newmark Method and the Space Discretization}

The global system to solve in the solid is expressed as following

$$
\begin{aligned}
\rho_{s} \mathbf{a}-\nabla \cdot \boldsymbol{\sigma}_{s}(\mathbf{u})-\rho_{s} \mathbf{b}_{s} & =0, \\
\mathbf{v} & =\dot{\mathbf{u}}, \\
\mathbf{a} & =\dot{\mathbf{v}} .
\end{aligned}
$$

The Newmark form taken by (18) in the particular case of the trapezoidale rule is

$$
\begin{aligned}
& H\left(\mathbf{a}_{n+1}\right)+G\left(\mathbf{u}_{n+1}\right)-F_{n+1}=0, \\
& \mathbf{u}_{n+1}=\mathbf{u}_{n}+\frac{1}{2} \tau\left(\mathbf{v}_{n}+\mathbf{v}_{n+1}\right), \\
& \mathbf{v}_{n+1}=\mathbf{v}_{n}+\frac{1}{2} \tau\left(\mathbf{a}_{n}+\mathbf{a}_{n+1}\right),
\end{aligned}
$$

where $\tau$ is the time step and $n$ the time level. $\mathrm{H}, \mathrm{G}$ and $F_{n+1}$ are respectively, the weak form of the term depending on the acceleration

$$
H\left(\mathbf{a}_{n+1}\right)=\int_{\Omega_{0}^{s}} \rho_{s} \mathbf{a}_{n+1} \cdot \boldsymbol{\Phi} d x
$$

the weak form of the strain tensor depending on the displacement

$$
G\left(\mathbf{u}_{n+1}\right)=\int_{\Omega^{s}} \sigma\left(\mathbf{u}_{n+1}\right): \nabla \boldsymbol{\Phi} d x
$$

and the weak form of the source term depending on the body force and the Neumann contribution $\mathbf{g}$ on the boundary with Neumann conditions $\Gamma_{N}$

$$
F_{n+1}=\int_{\Omega^{s}} \rho_{s} \mathbf{b}_{s} \cdot \boldsymbol{\Phi} d x+\int_{\Gamma_{N}} \mathbf{g} \cdot \boldsymbol{\Phi} d s .
$$

This method is second-order accurate in time.

We transform again (19) in order to solve a predictor-corrector algorithm in the same way as [18]. At each time level $n$, we begin with a prediction on the three kinematic fields to compute

$$
\begin{aligned}
\mathbf{u}_{n+1}^{p} & =\mathbf{u}_{n}+\tau \mathbf{v}_{n}+\frac{\tau^{2}}{2} \mathbf{a}_{n}, \\
\mathbf{v}_{n+1}^{p} & =\mathbf{v}_{n}+\tau \mathbf{a}_{n}, \\
\mathbf{a}_{n+1}^{p} & =\mathbf{a}_{n} .
\end{aligned}
$$

To form the final system, we introduce three corrector fields denoted by $\delta \mathbf{u}, \delta \mathbf{v}$, and $\delta \mathbf{a}$ and defined as

$$
\begin{aligned}
& \delta \mathbf{u}=\mathbf{u}_{n+1}-\mathbf{u}_{n+1}^{p}, \\
& \delta \mathbf{v}=\mathbf{v}_{n+1}-\mathbf{v}_{n+1}^{p}, \\
& \delta \mathbf{a}=\mathbf{a}_{n+1}-\mathbf{a}_{n+1}^{p} .
\end{aligned}
$$


Now, let us replace the predictor fields in (24) by their expression in (23), we have

$$
\begin{aligned}
& \delta \mathbf{u}=\mathbf{u}_{n+1}-\mathbf{u}_{n}-\tau \mathbf{v}_{n}-\frac{\tau^{2}}{2} \mathbf{a}_{n}, \\
& \delta \mathbf{v}=\mathbf{v}_{n+1}-\mathbf{v}_{n}-\tau \mathbf{a}_{n}, \\
& \delta \mathbf{a}=\mathbf{a}_{n+1}-\mathbf{a}_{n} .
\end{aligned}
$$

Therefore plugging the two last equations of (19) in (25) yields

$$
\begin{aligned}
\left\{\begin{array}{l}
\delta \mathbf{u}= \\
\delta \mathbf{v}=\frac{1}{2} \tau\left(\mathbf{v}_{n}+\mathbf{v}_{n+1}\right)-\tau\left(\mathbf{v}_{n}-\frac{\tau^{2}}{2} \mathbf{a}_{n}+\mathbf{a}_{n+1}\right)-\tau \mathbf{a}_{n} \\
\delta \mathbf{a}=\mathbf{a}_{n+1}-\mathbf{a}_{n}
\end{array}\right. \\
\qquad\left\{\begin{array} { l } 
{ \delta \mathbf { u } = \frac { 1 } { 2 } \tau ( \frac { 1 } { 2 } \tau ( \mathbf { a } _ { n } + \mathbf { a } _ { n + 1 } ) ) - \frac { \tau ^ { 2 } } { 2 } \mathbf { a } _ { n } } \\
{ \delta \mathbf { u } = \frac { 1 } { 2 } \tau ( \mathbf { v } _ { n + 1 } - \mathbf { v } _ { n } ) - \frac { \tau ^ { 2 } } { 2 } \mathbf { a } _ { n } } \\
{ \delta \mathbf { v } = \frac { 1 } { 2 } \tau ( \mathbf { a } _ { n + 1 } - \mathbf { a } _ { n } ) } \\
{ \delta \mathbf { a } = \frac { 1 } { 2 } \tau ( \mathbf { a } _ { n + 1 } - \mathbf { a } _ { n } ) }
\end{array} \Longleftrightarrow \left\{\begin{array}{l}
\delta \mathbf{u}=\frac{1}{4} \tau{ }^{2} \delta \mathbf{a}, \\
\delta \mathbf{v}=\frac{1}{2} \tau \delta \mathbf{a}, \\
\delta \mathbf{a}=\mathbf{a}_{n+1}-\mathbf{a}_{n} .
\end{array}\right.\right.
\end{aligned}
$$

We deduce the link between $\delta \mathbf{v}, \delta \mathbf{a}$, and $\delta \mathbf{u}$

$$
\begin{aligned}
& \delta \mathbf{v}=\frac{2 \delta \mathbf{u}}{\tau}, \\
& \delta \mathbf{a}=\frac{4 \delta \mathbf{u}}{\tau^{2}} .
\end{aligned}
$$

Finally, we replace these last relations in the first equation of (19) to form the variational formulation on $\delta \mathbf{u} \in \mathbf{V}=\left\{\mathbf{v} \in H^{1}\left(\Omega^{s}\right)^{d}:\left.\mathbf{v}\right|_{\Gamma}\right.$ given $\}$ and using $\boldsymbol{\Phi} \in \mathbf{V}_{0}=\left\{\mathbf{v} \in H^{1}\left(\Omega^{s}\right)^{d}:\left.\mathbf{v}\right|_{\Gamma}=0\right\}$ as test function where $\Gamma$ is the boundary with Dirichlet conditions

$$
\begin{aligned}
& \frac{4 \rho_{s}}{\tau^{2}} \int_{\Omega^{s}} \delta \mathbf{u} \cdot \boldsymbol{\Phi} d x+2 \mu \int_{\Omega^{s}} \boldsymbol{\varepsilon}(\delta \mathbf{u}): \boldsymbol{\varepsilon}(\boldsymbol{\Phi}) d x \\
& \quad+\lambda \int_{\Omega^{s}}(\nabla \cdot \delta \mathbf{u})(\nabla \cdot \boldsymbol{\Phi}) d x-\langle\mathbf{F}, \boldsymbol{\Phi}\rangle=0, \quad \forall \boldsymbol{\Phi} \in \mathbf{V}_{0},
\end{aligned}
$$

where $\langle\mathbf{F}, \boldsymbol{\Phi}\rangle=\int_{\Omega^{s}} \rho_{s}\left(\mathbf{b}_{s}-\mathbf{a}_{n+1}^{p}\right) \cdot \boldsymbol{\Phi} d x-\int_{\Omega^{s}} \boldsymbol{\sigma}\left(\mathbf{u}_{n+1}^{p}\right): \nabla \boldsymbol{\Phi} d x+\int_{\Gamma_{N}} \mathbf{g} \cdot \boldsymbol{\Phi} d s$. The addition of the corrections to the predicted fields gives the new fields at step $n+1$ according to (24). Before beginning the Newmark solution we compute the initial acceleration

$$
\int_{\Omega_{0}^{s}} \rho_{s} \mathbf{a}_{0} \cdot \boldsymbol{\Phi} d x=\int_{\Omega_{0}^{s}} \rho_{s} \mathbf{b}_{s} \cdot \boldsymbol{\Phi} d x+\int_{\Gamma_{N}} \mathbf{g} \cdot \boldsymbol{\Phi} d s-\int_{\Omega_{0}^{s}} \boldsymbol{\sigma}\left(\mathbf{u}_{0}\right): \nabla \boldsymbol{\Phi} d x
$$

with Dirichlet conditions. 
In the case of the fluid-structure interaction we want to emphasize how the integral in the natural boundary has to be understood

$$
\int_{\Gamma_{N}} \mathbf{g} \cdot \boldsymbol{\Phi} d s=\int_{\Gamma_{N} / \Gamma_{I}} \mathbf{g} \cdot \boldsymbol{\Phi} d s+\int_{\Gamma_{I}} \mathbf{g} \cdot \boldsymbol{\Phi} d s
$$

with the notation $\Gamma_{I}$ for the interface. Frequently on $\Gamma_{N} / \Gamma_{I}$, we will assume $\mathbf{g}=0$. On $\Gamma_{I}$, we will impose the Neumann condition

$$
\int_{\Gamma_{I}} \mathbf{g} \cdot \boldsymbol{\Phi} d s=\int_{\Gamma_{I}} \boldsymbol{\sigma}_{s} \mathbf{n} \cdot \boldsymbol{\Phi} d s=-\int_{\Gamma_{I}} \boldsymbol{\sigma}_{f} \mathbf{n} \cdot \boldsymbol{\Phi} d s
$$

This equation produces a relation that links the fluid and the structure at the interface.

The space discretization of the variables $\mathbf{u}, \mathbf{v}$, and $\mathbf{a}$ is made in the $P_{N}$ space. This choice for these fields is consistent from the mechanical point of view with that of the fluid velocity and allows an easy communication between the fluid and solid parts of the problem.

\section{FLUID-STRUCTURE CALCULATION}

\subsection{Fluid-Structure System}

The complete system to solve the fluid-structure interaction can be summarized in the fluid domain $\Omega^{f}$ by Eqs. (1) and (15) in the structure. At the interface $\Gamma_{I}$, we solve the velocity continuity and the equality of the contact forces

$$
\text { (I) }\left\{\begin{aligned}
\sigma_{f} \mathbf{n} & =\sigma_{s} \mathbf{n} \\
\mathbf{v} & =\dot{\mathbf{u}} .
\end{aligned}\right.
$$

We have solved this problem considering in the fluid domain the motion due to the structure. We move each node of the grid and compute the Jacobian and the inverse of the Jacobian matrix on the deformed elements. No function was directly available to modify the mesh at each time step $[9,16]$. Thus, we have modified some function of SPECULOOS [19] which was only able to generate the grid in relation with the initial fixed nodes. Henceforth, SPECULOOS is able to take into account the motion of the mesh only modifying the values of the coordinates. The Jacobian and the inverse of the Jacobian matrix are computed automatically with these new coordinates. 


\subsection{ALE Field Computation}

When the structure is deformed, the fluid domain undergoes the same deformation. We assume that a portion of the fluid domain remains fixed and another part can have a motion computed with an elliptic system to obtain a moving field. This grid moves at each time step and brings the field velocity mesh $\mathbf{w}$ as in Eqs. (1) and (8). Therefore we solve a Laplacian or an elastostatic operator as in $\mathrm{Ho}[8]$

$$
\nabla_{\mathbf{X}} \cdot \tilde{\boldsymbol{\sigma}}(\mathbf{w})=0,
$$

where appropriate boundary conditions for the stress are imposed. Knowing the velocity field $\mathbf{w}$, we can deduce the new ALE grid in the fluid domain by solving (7). To update the ALE grid, we use the second-order time accurate trapezoidal rule

$$
\boldsymbol{x}^{n+1}=\boldsymbol{x}^{n}+\frac{\tau}{2}\left(\mathbf{w}^{n+1}+\mathbf{w}^{n}\right) .
$$

If we note by $\boldsymbol{x}_{s}$ the coordinate field in the structure, the structural mesh can be updated with

$$
\boldsymbol{x}_{s}^{n+1}=\boldsymbol{x}_{s}^{n}+\mathbf{u}^{n+1} .
$$

In this paper we consider that the structure position is updated to the step $n+1$ independently of the $n+1$ fluid step. Other methods resort to a mesh

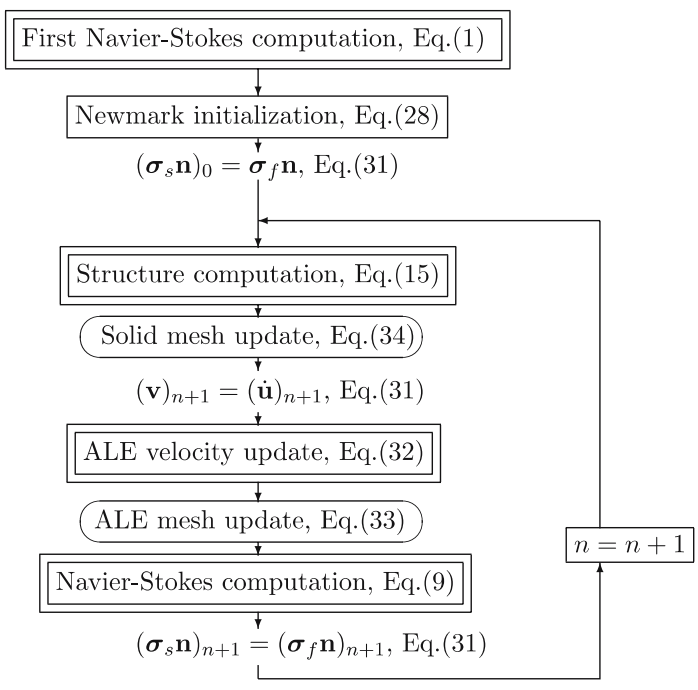

Fig. 2. Fluid-structure algorithm. 
update by subcycling the fluid integration to compute a better correction of the mesh structure in order to improve the geometric conservation law or to adapt the improved serial staggered (ISS) procedure [20] in order to obtain a second-order time-accurate system [13].

\subsection{The General Algorithm}

The FSI algorithm works as follows. In a first step, we initialize the Newmark method through (28). Then, we compute a first stationary solution of the Navier-stokes problem keeping the structure fixed. The fluid part solution sends a Neumann condition to the structure which allows to define the new ALE velocity $\mathbf{w}$ and its associated mesh. So we know the new velocity Dirichlet condition to impose on the fluid at $\Gamma_{I}$. This algorithm is summarized in Fig. 2.
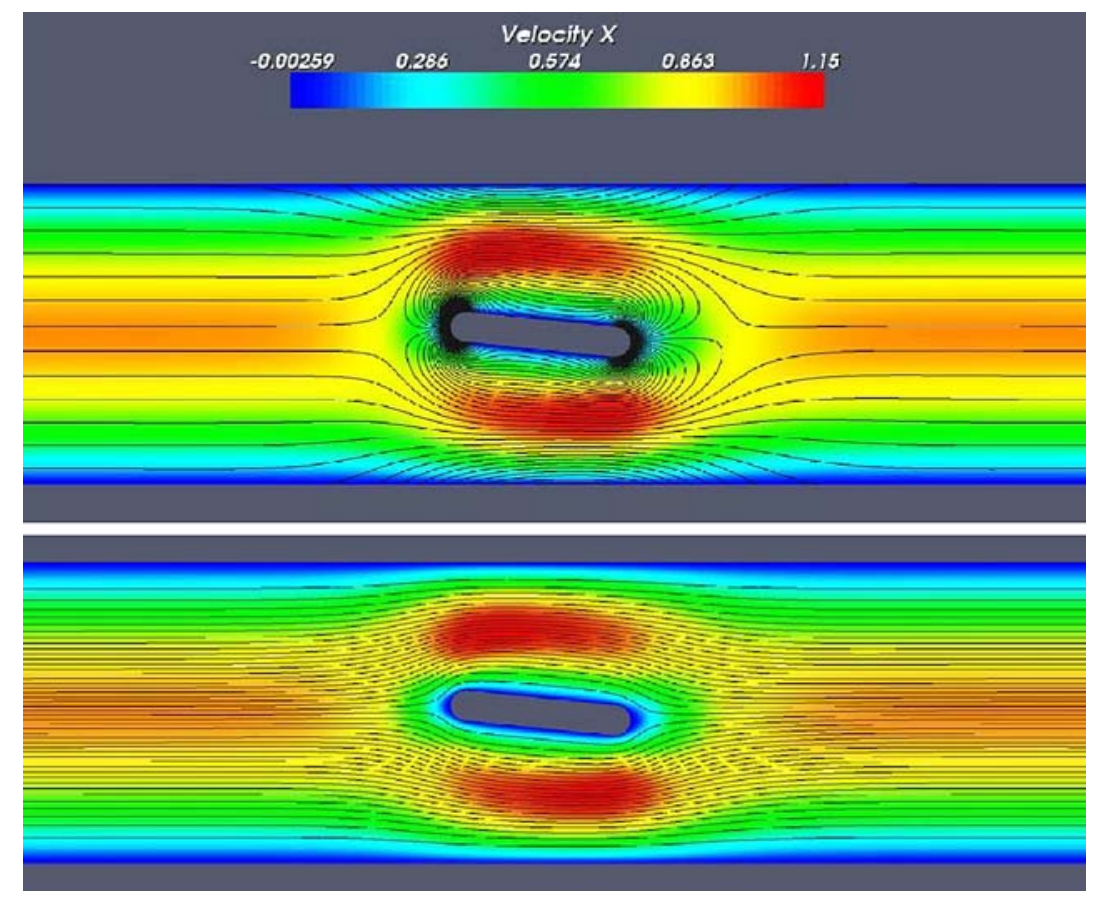

Fig. 3. The $v_{x}$ field with isolines of the vorticity $\omega$ (top) and stream function $\Psi$ (bottom) at $t=10^{-2}$. 


\section{NUMERICAL RESULTS}

\subsection{Visualization of Simulations}

We impose a steady-state Poiseuille flow at the entry for a Reynolds number $R e=10$. In order to avoid a symmetric solution, we disturb the initial position of the structure by titling it with an angle of $0.05 \mathrm{rad}$. The calculation is performed on a cluster of Pentium IV PCs using eight processors during for about 60 hours. The fluid mesh contains 2184 $(\sim 42 \times 60)$ elements, the structure mesh $620(\sim 38 \times 18)$ and we have chosen $N=4$ as polynomial degree in each direction. We show two solutions time steps of the problem with $\tau=10^{-3}$ at times $t=10^{-2}$ (Fig. 3) and $t=310^{-1}$ (Fig. 4). These figures display the $v_{x}$ velocity field with the associated isolines of the vorticity $\omega=$ curl $\mathbf{v}$ and the stream function $\Psi$.
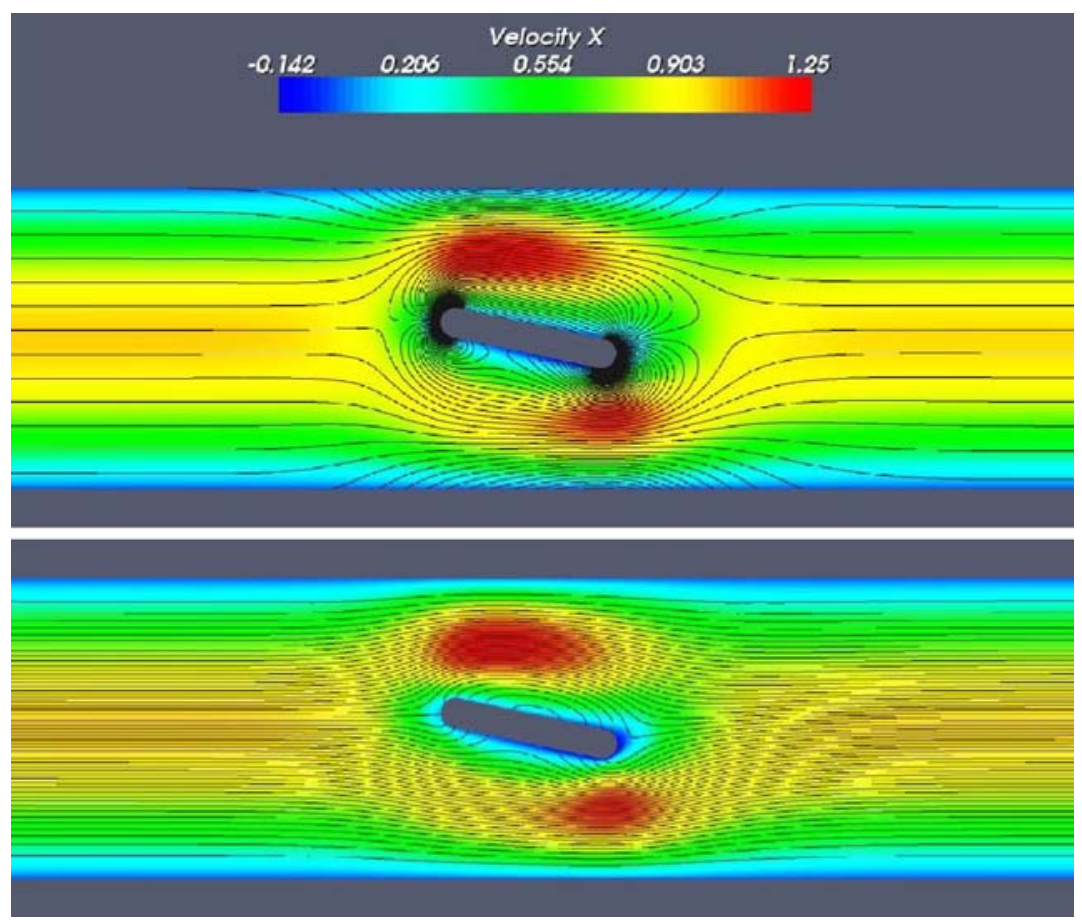

Fig. 4. The $v_{x}$ field with isolines of the vorticity $\omega$ (top) and stream function $\Psi$ (bottom) at $t=3 \times 10^{-1}$. 


\subsection{Norm Analysis of the Solution}

We have computed the following expression $\frac{\left\|\mathbf{v}_{n+1}-\mathbf{v}_{n}\right\|_{L^{2}}}{\tau}$ as an approximation of the acceleration norm on the fluid domain to check the evolution of the non-stationary problem when the structure stays fixed during the first 1000 time steps (top) and subsequently when the structure is in motion (bottom)(Fig. 5).

The top curve of the Fig. 5 shows the fluid flow reaches the stationary state while in the next sequence the flow goes away from it when the structure inside the channel is allowed to move. We have also compared the norm of the acceleration when the time step is changed to $\tau=2.5 \times$ $10^{-3}$ and we have compared the computations until $t=2.5 \times 10^{-1}$. One observes a discrepancy between the two curves due to the fact the computation began with different stationary states as exhibited in Fig. 6. Nevertheless, the evolution in the next time steps is similar.
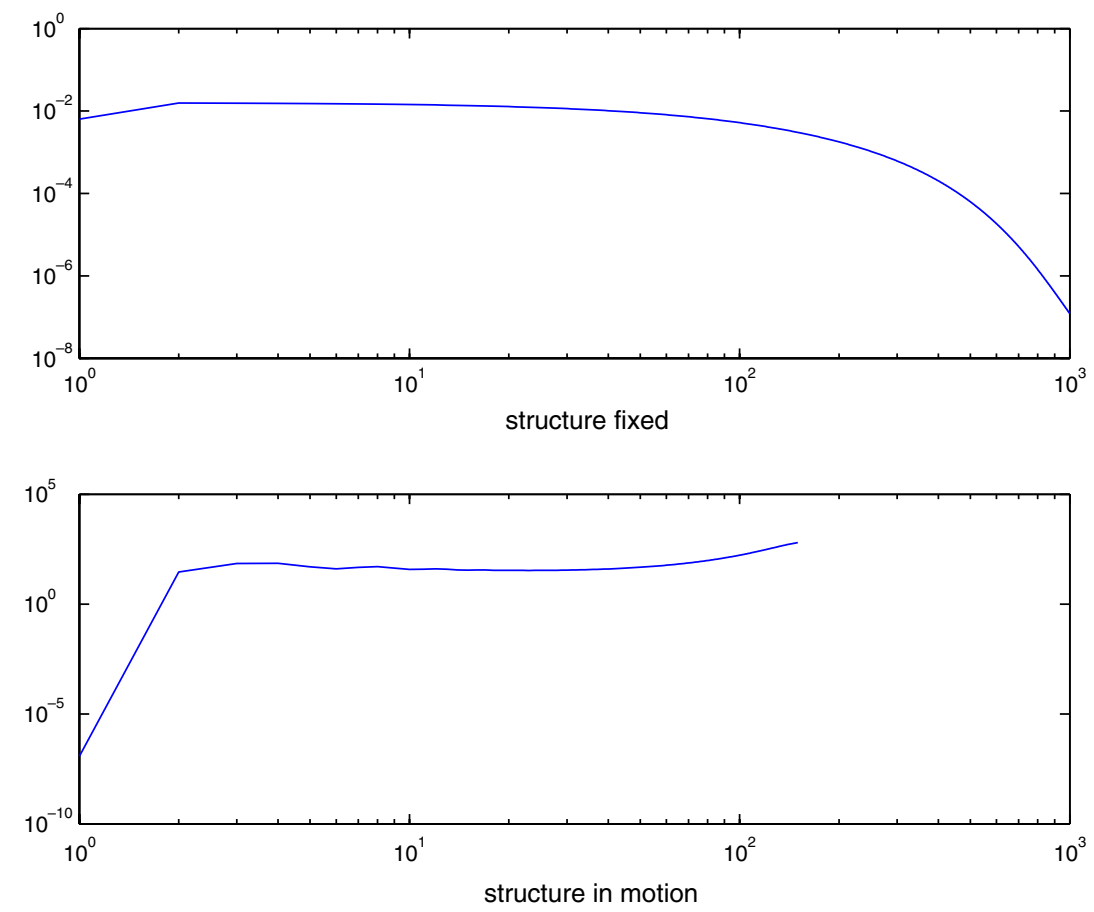

Fig. 5. $\frac{\left\|\mathbf{v}_{n+1}-\mathbf{v}_{n}\right\|_{L^{2}}}{\tau}$ versus time. 


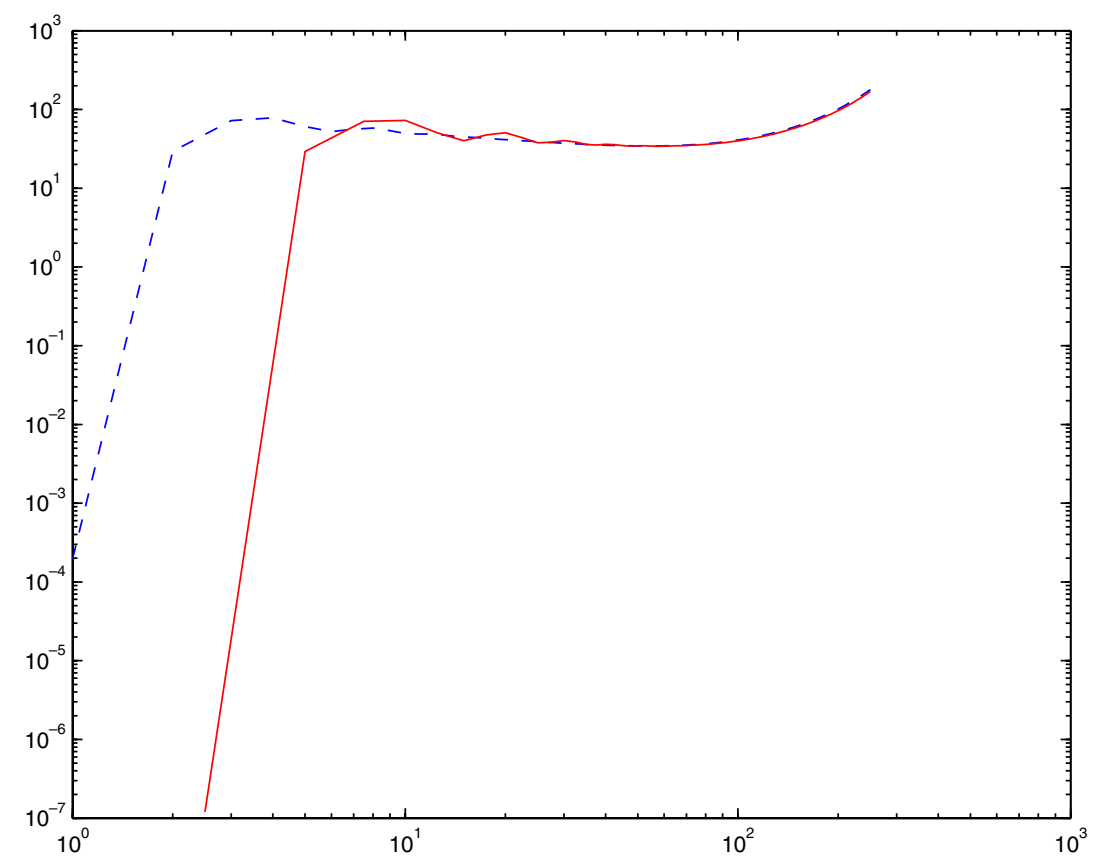

Fig. 6. Comparison of $\frac{\left\|\mathbf{v}_{n+1}-\mathbf{v}_{n}\right\|_{L^{2}}}{\tau}$ with respect to the time step between the case $\tau=10^{-3}$ (dashed) and the case $\tau=2.5 \times 10^{-3}$ (full).

\section{CONCLUSIONS}

In this paper, we presented a numerical method based on SEM and second-order time marching schemes for fluid and solid domains, respectively. The FSI is segregated in the time scheme. The solution of a channel flow with the presence of a solid object moving under the action of the fluid has demonstrated the feasibiliy of the method.

\section{REFERENCES}

1. Formaggia, L., Gerbeau, J.-F., Nobile, F., and Quarteroni, A. (2001). On the coupling of 3D and 1D Navier-Stokes equations for flow problems in compliant vessels. Comput. Meth. Appl. Mech. Engrg. 191, 561-582.

2. Le Tallec, P., and Mouro, J. (2001). Fluid-structure interaction with large structural displacement. Comput. Meth. Appl. Mech. Engrg. 190, 3039-3067.

3. Ramaswamy, B., and Kawahara, M. (1987). Arbitrary Lagrangian-Eulerian finite element method for unsteady, convective, incompressible viscous free surface fluid flow. In Gallagher, R. H., Glowinsky, R., Gresho, P. M., Oden J. T., and Zienkiewicz, O. C. (eds.), Finite Elements in Fluids-Volume 7, John Wiley and Sons Ltd., New York, pp. 65-87. 
4. Blom, F. (1998). Investigation on computational fluid-structure interaction, Ph.D. n 1865, EPFL, Lausanne.

5. Glowinski, R., Pan, T.-W., and Periaux, J. (1994). A ficticious domain method for Dirichlet problem and applications. Comput. Meth. Appl. Mech. Engrg. 111, 283-303.

6. De Hart, J., Peters, G. W. M., Schreurs, P. J. G., and Baaijens, F. P. T. (2000). A two-dimensional fluid-structure interaction model of the aortic valve. J. Biomech. 33, 1079-1088.

7. Causin, P., Gerbeau, J. F., and Nobile F., (2005). Added-mass effect in the design of partitioned algorithms for fluid-structure problems. Comput. Methods Appl. Mech. Engrg. 193, 4073-4095.

8. Ho, L.-W., and Patera, A. T. (1990). A Legendre Element Method for Simulation of Incompressible Unsteady Viscous Free-surface Flows. Comput. Methods Appl. Mech. Engrg. 80, 355-366.

9. Deville, M. O., Fischer, P. F., and Mund, E. H. (2002). High-Order Methods for Incompressible Fluid Flow, Cambridge University Press, Cambridge.

10. Widlund, O. B., and Pavarino, L. F. (1999). Iterative substructuring methods for spectral element discretizations of elliptic systems.II: mixed methods for linear elasticity and Stokes flow. SIAM J. Numer. Anal. 37(2), 375-402.

11. Casadei, F., Gabellini, E., Fotia, G., Maggio, F., and Quarteroni, A. (1993). A mortar spectral/finite element method for complex 2D and 3D elastodynamic problems. Comput. Meth. Appl. Mech. Engrg. 104, 49-76.

12. Donea, J., Guiliani, S., and Halleux, J. P. (1982). An arbitrary Lagrangian-Eulerian finite element method for transient dynamic fluid-structure interactions. Comput. Meth. Appl. Mech. Engrg. 33, 689-723.

13. Formaggia, L., and Nobile, F. (2004). Stability analysis of second-order time accurate schemes for ALE-FEM. Comput. Methods Appl. Mech. Engrg. 193, 4097-4116.

14. Boffi, D., and Gastaldi, L. (2004). Stability and geometric conservation laws for ALE formulations. Comput. Methods Appl. Mech. Engrg. 193, 4717-4739.

15. Maday, Y., and Patera, A. T. (1989). Spectral element methods for the Navier-Stokes equations. In Noor, A. K., and Oden, J. T. (eds.), State-of-the-Art Surveys in Computational Mechanics, ASME, New York, pp. 71-143.

16. Couzy, W., and Deville, M. O. (1994). Spectral-element preconditoners for the Uzawa pressure operator applied to incompressible flows. J. Sci. Comput. 9, 107-112.

17. Perot, J. B. (1993). An analysis of the fractional step method. J. Comp. Phys. 108, 51-58.

18. Curnier, A. (1993). Méthodes numériques en mécanique des solides, Presses polytechniques et universitaires romandes, Lausanne.

19. Dubois-Pèlerin, Y., Van Kemenade, V., and Deville, M. O. (1999). An Object-Oriented Toolbox for Spectral Element Analysis. J. Sci. Comput. 14, 1-29.

20. Farhat, C., and Lesoinne, M. (2000). Two efficient staggered algorithms for serial and parallel solution of three-dimensional nonlinear transient aeroelastic problems. Comput. Meth. Appl. Mech. Engrg. 182, 499-515. 